\title{
Feasibility of Terahertz Time-Domain Spectroscopy to Detect Carbendazim Mixtures Wrapped in Paper
}

\author{
Binyi Qin, ${ }^{1,2}$ Zhi Li, ${ }^{1,3}$ Zhihui Luo, ${ }^{4}$ Huo Zhang, and Yun $\mathrm{Li}^{4}$ \\ ${ }^{1}$ School of Mechano-Electronic Engineering, Xidian University, Xi'an, Shanxi 710126, China \\ ${ }^{2}$ School of Electronics and Communication Engineering, Yulin Normal University, Yulin, Guangxi 537000, China \\ ${ }^{3}$ Guangxi Key Laboratory of Automatic Detecting Technology and Instruments, School of Electronic Engineering and Automation, \\ Guilin University of Electronic Technology, Guilin, Guangxi 541004, China \\ ${ }^{4}$ Guangxi Key Laboratory for Agricultural Resources Chemistry and Efficient Utilization (Cultivation Base), Colleges and Universities \\ Key Laboratory for Efficient Use of Agricultural Resources in the Southeast of Guangxi, College of Chemistry and Food Science, \\ Yulin Normal University, Yulin, Guangxi 537000, China
}

Correspondence should be addressed to Zhi Li; zhili_workemail@163.com

Received 9 March 2017; Accepted 13 July 2017; Published 9 August 2017

Academic Editor: Khalique Ahmed

Copyright ( 2017 Binyi Qin et al. This is an open access article distributed under the Creative Commons Attribution License, which permits unrestricted use, distribution, and reproduction in any medium, provided the original work is properly cited.

\begin{abstract}
The purpose of this work was to detect carbendazim mixtures wrapped in paper. Unlike previous reports of THz-TDS for detecting pesticide residue, this work focused on detecting pesticide residue in packaged foods. Different weight ratios of carbendazim in polyethylene and in rice powder were detected qualitatively and quantitatively. Results show that pure carbendazim, polyethylene, and rice powder can be easily distinguished from each other. However, when the weight ratio was low, the absorbance of the mixture was similar with that of pure polyethylene and rice powder. With the help of SVM, carbendazim could be qualitatively detected in low weight ratio mixture successfully. Moreover, PLS and SVR were selected to quantitatively detect carbendazim mixtures. SVR has higher R and lower RMSECV, RMSEC, and RMSEP than PLS model. Lasty, the results also indicate that THz-TDS is a potential tool to detect pesticide residue in packaged foods qualitatively and quantitatively.
\end{abstract}

\section{Introduction}

Pesticide has been widely used in agriculture due to its broad spectrum activity and low production costs. However, overusing pesticide has cased residue of this drug in food product, which is detrimental to the health of consumers. Thus, detecting pesticide residue has become more and more important in the modern world. Traditional methods of pesticide residue detection include gas chromatography, liquid chromatography, and high-performance liquid chromatography. Nevertheless, these methods are time consuming and require tedious sample preparation procedures and cannot offer fast pesticide detection [1-3]. Other rapid detection methods based on immunology assays, such as immunochemistry and biosensors are time saving, sensitive, and highly selective, but they are difficult to establish and usually yield false-positive results [4].

In recent years, spectroscopic methods, such as ultraviolet spectroscopy, near-infrared spectroscopy, and infrared spectroscopy, are applied to detect pesticide residue because of their nondestructive and simple sample pretreatment. But there are limitations to these methods. Ultraviolet spectroscopy is harmful to the active ingredients in food and the human body [5]. Near-infrared spectroscopy is only suitable for the quantitative analysis rather than the qualitative analysis, owing to its complex spectra for intramolecular overtone and combination bands of chemicals [6]. In contrast, infrared spectroscopy is used for a qualitative tool rather than as a quantitative tool as a result of its sensitivity to the scattering effect in the sample and less stable radiation 


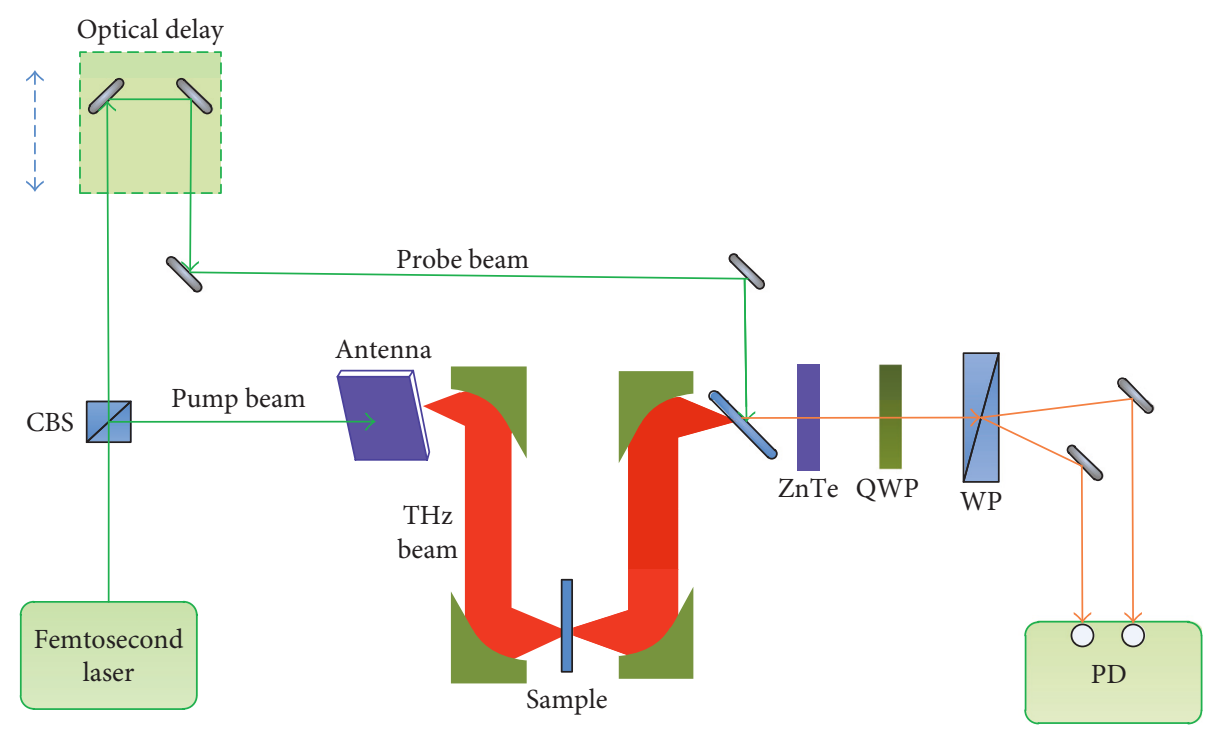

FIGURE 1: Schematic of the experimental apparatus.

source [7]. Therefore, there is an increasing demand to exploit new techniques for rapid, sensitive, and nondestructive detection of pesticide residue in routine assays.

Terahertz (THz) refers to the electromagnetic wave in the frequency range of 0.1 to $10 \mathrm{THz}$ between microwave and infrared. Comparing with other spectroscopic methods, $\mathrm{THz}$ has little damage to the target material because of its low photon energy and is less sensitive to noise that comes from thermal background radiation [8]. Moreover, it can give the amplitude and phase information of the sample simultaneously. So, $\mathrm{THz}$ has been qualitatively and quantitatively applied in many fields, for example chemistry, biology, medical science, and homeland security [9-13].

In the field of pesticide detection, Bing-Hua et al. analyzed dimethoate using density functional theory and experimental investigations [14]. Hua et al. quantitatively studied cyfluthrin $\mathrm{n}$-hexane solutions based on partial least squares (PLS) and principal component regression (PCR) methods [15]. Qiang et al. used partial least squares (PLS), interval PLS (iPLS), moving window PLS (mwPLS), and backward interval PLS (biPLS) methods to quantify nitrofen [16]. However, few papers are concerned for detecting pesticide residue in packaged foods. It will be a meaningful progress.

In this work, we mixed carbendazim with polyethylene and rice powder, separately, and then employed terahertz time-domain spectroscopy (THz-TDS) to analyze these mixtures wrapped in paper. Firstly, we observed the absorbency of carbendazim in $\mathrm{THz}$ range. Secondly, we analyzed the absorbency of mixtures wrapped in paper. And then, the absorbency of the $\mathrm{THz}$ spectrum was used as input of support vector machine (SVM), partial least squares regression (PLSR), and support vector regression (SVR) for qualitative and quantitative analysis. The results indicate that pesticide can be qualitatively and quantitatively detected in mixtures and give evidence of a potential of applying $\mathrm{THz}$-TDS to detect the presence of pesticide in packaged foods.

\section{Materials and Methods}

2.1. Experimental System. The experimental apparatus of the terahertz time-domain spectroscopy (THz-TDS) system, shown in Figure 1, is composed of two parts: a terahertz time-domain spectrometer Z-3 (Zomega Terahertz Corp., USA) and an ultrafast fiber laser FemtoFiber pro NIR (TOPTICA Photonics Inc., Germany). The ultrafast fiber laser generates a laser beam, with a pulse width of about $100 \mathrm{fs}$, the wavelength centered around $780 \mathrm{~nm}$, the repetition rate of about $80 \mathrm{MHz}$, and the average power of nearly $100 \mathrm{~mW}$. The laser beam is split into a pump beam and a probe beam by a cubic beam splitter (CBS) for THz generation and detection, respectively. The pump beam elicits the terahertz beam at the emitter, which is composed of a photoconductive antenna. Then, the generated $\mathrm{THz}$ beam is focused onto the sample, carries sample characteristics, and meets the probe beam at the $\mathrm{ZnTe}$ detector, where the probe beam is modulated by $\mathrm{THz}$ radiation through the electro-optic effect. After transmitting through a quater-wave-plate (QWP) and a Wollaston prism (WP), the modulated probe beam is then detected by a set of balanced photodiodes (PD).

In the experiment, we utilized the THz-TDS system in transmission mode. In order to reduce absorption of $\mathrm{THz}$ spectra by atmospheric water vapor, the $\mathrm{THz}$ beam path was enclosed in a box and dry air was injected into the box. During experiment, the relative humidity within the box was less than $4 \%$, and the temperature was kept at room temperature (about $295 \mathrm{~K}$ ).

2.2. Sample Preparation. Carbendazim powder with a purity of $98 \%$ was purchased from Adamas Company. High-density polyethylene powder was supplied by the Sigma-Aldrich company. Rice powder was obtained from a local market. To remove the water, all the powder-form samples were dried in a vacuum-drying oven for one hour at $323 \mathrm{~K}$. 
A series of mixtures with weight ratios ranging from $0 \%$ to $100 \%$ were prepared by mixing appropriate amounts of carbendazim in polyethylene and rice powder. And 25 replicates were prepared for each weight ratio. All the mixtures were mixed and ground by using a pestle and mortar, filtrated by a 200-mesh sieve, and then pressed into a $1-1.4 \mathrm{~mm}$ thick disk by a tablet press.

2.3. Data Acquisition. THz waveform was measured directly by $\mathrm{THz}$-TDS system. Both reference and sample $\mathrm{THz}$ time-domain spectra were obtained from free path and sample measurement. To avoid echoing influence, the time-domain waveform was truncated firstly. And then, a fast Fourier transform was applied to obtain the spectral distribution in a frequency domain from time-domain $\mathrm{THz}$ waveform. After that, the absorbance spectrum could be acquired as follows [17]:

$$
\operatorname{Absorbance}(\omega)=-\log _{10}\left|\frac{E_{\mathrm{s}}(\omega)}{E_{\text {ref }}(\omega)}\right|^{2},
$$

where $E_{s}(\omega)$ and $E_{\text {ref }}(\omega)$ were the amplitude of the sample and reference signal in a frequency domain, respectively.

In the experiment, we obtained the absorbance spectrum of pure carbendazim firstly. After that, the absorbance spectrums of mixtures, pure carbendazim, pure rice powder, and pure polyethylene, which were wrapped in paper, were gained.

2.4. Qualitative Modeling and Evaluation. With qualitative detection, SVM was employed. It is a supervised learning model with associated learning algorithms that analyze data used for classification. The SVM model maps the instances into a space, in which the instances of the categories are separated by a distinct gap that is as wide as possible. And then, new instances are mapped into the same space and predicted to belong to a category based on which side of the gap they fall $[18,19]$. In addition, SVM can efficiently perform a nonlinear classification using the kernel trick.

Accuracy of cross validation (ACCCV) set was applied to optimize the model parameters. Accuracy of training (ACCTR) set was applied to evaluate the qualitative model. Accuracy of testing (ACCTE) set was used to evaluate generalization ability of the qualitative model. Accuracy is calculated using

$$
\text { accuracy }=\frac{\text { correctly predicted data }}{\text { total testing data }} \times 100 \% \text {. }
$$

Generally, a good model should have higher ACCCV, ACCTR, and ACCTE.

2.5. Quantitative Modeling and Evaluation. PLS is a linear regression method, able to deal with collinear variables and accepting a large number of variables. It linearly projects both input data and output data into the subspace, to find a feature matrix that can best represent the input data with as much variance as possible while correlating to the output to the maximum extent $[20,21]$. Thus, the feature matrix

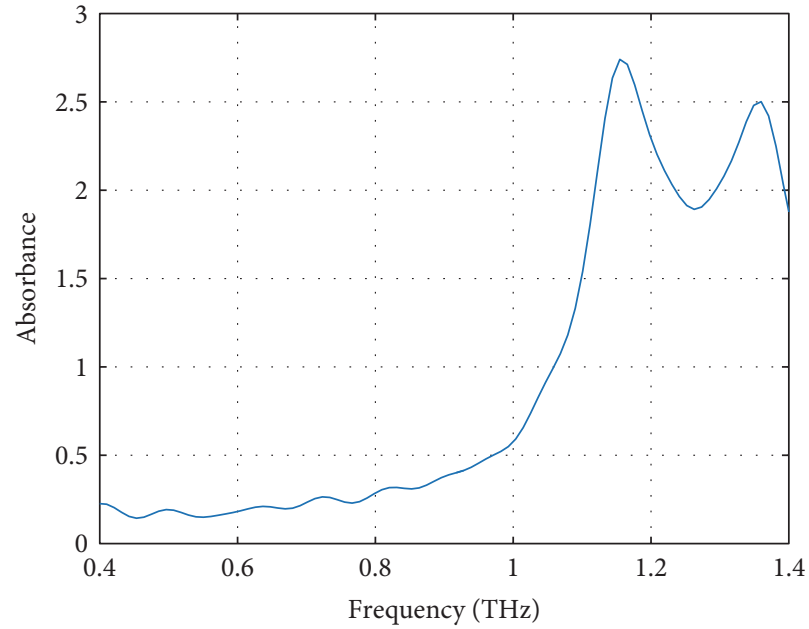

FIgURE 2: Average absorbance spectrum of pure carbendazim.

carries the most useful information of input data, which are correlated to output data.

SVR is a nonlinear regression method, constructed by employing the $\varepsilon$-insensitive loss function, the penalty factor $C$, and the kernel function of radial basis function (RBF). The $\varepsilon$-insensitive loss function determines a deviation of $\pm \varepsilon$. Instances, those prediction errors that exceed the given deviation of $\pm \varepsilon$, would be penalized by the penalty factor $C$ $[22,23]$. In the experiment, $\varepsilon$ was equal to 0.001 . The kernel function is a nonlinear mapping function, which maps instances to a hyperdimensional feature space where the solution may become linear.

The root mean square error of cross validation (RMSECV) set was applied to optimize the model parameters. The root mean square error of training (RMSEC) set was used to evaluate the quantitative model. The root mean square error of testing (RMSEP) set and the correlation coefficient $(R)$ were used to evaluate generalization ability of the quantitative model. RMSE and R are calculated using

$$
\begin{aligned}
\text { RMSE } & =\sqrt{\frac{\sum_{i=1}^{N}\left(y_{i}^{\mathrm{pre}}-y_{i}\right)^{2}}{N},} \\
R & =\frac{\sum_{i=1}^{N}\left(y_{i}-\bar{y}\right)\left(y_{i}^{\mathrm{pre}}-\bar{y}^{\mathrm{pre}}\right)}{\sqrt{\sum_{i=1}^{N}\left(y_{i}-\bar{y}\right)^{2} \sum_{i=1}^{N}\left(y_{i}^{\mathrm{pre}}-\bar{y}^{\mathrm{pre}}\right)^{2}}},
\end{aligned}
$$

where $N$ is the sample size, $y_{i}$ is actual value of the $i$ th sample, $y_{i}^{\text {pre }}$ is the predicted value of the $i$ th sample, $\bar{y}$ is the average of the sample actual value, and $\bar{y}^{\text {pre }}$ is the average of the sample predicted value.

A good model had a great model precision and prediction precision with higher $R$ and lower RMSECV, RMSEC, and RMSEP sets.

\section{Results and Discussions}

3.1. THz Spectra of Carbendazim Mixtures. Figure 2 shows the average absorbance spectrum of pure carbendazim. Carbendazim exhibited two relatively distinct absorption 


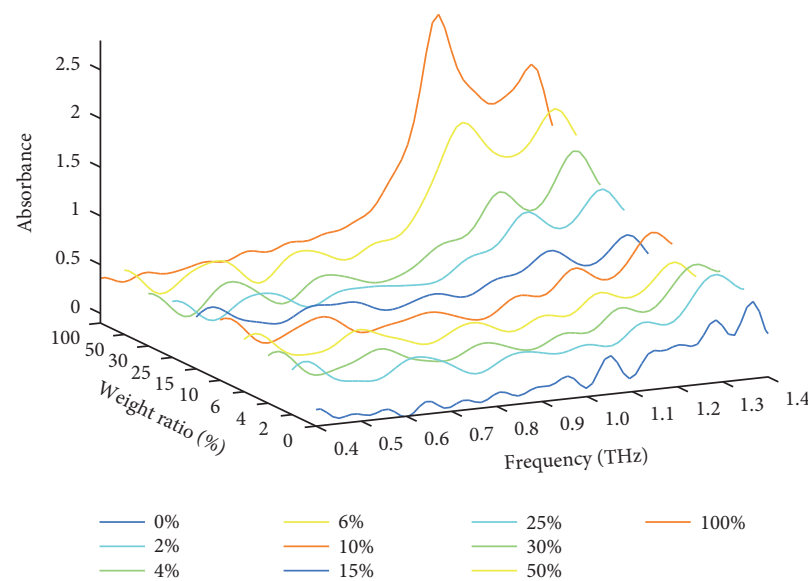

(a)

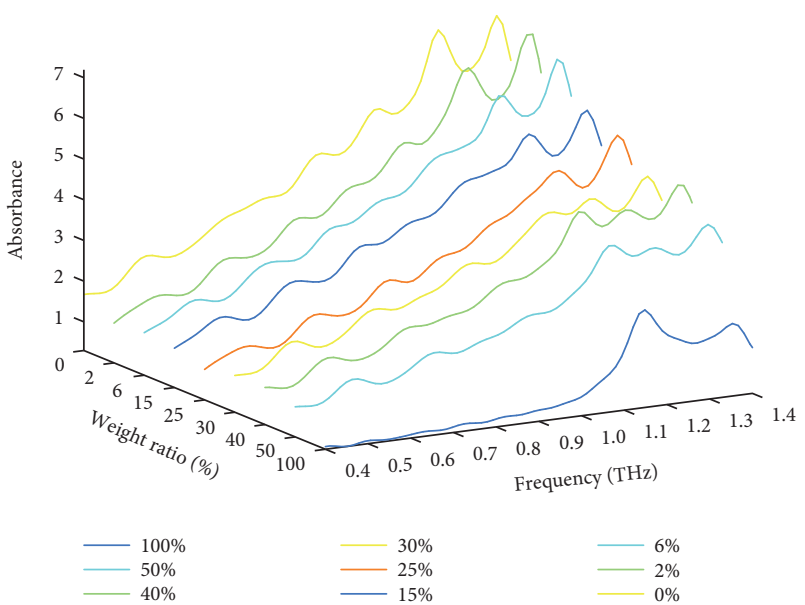

(b)

FIgURe 3: Average absorbance spectrum of mixtures wrapped in paper. (a) Carbendazim and polyethylene mixture. (b) Carbendazim and rice powder mixture.

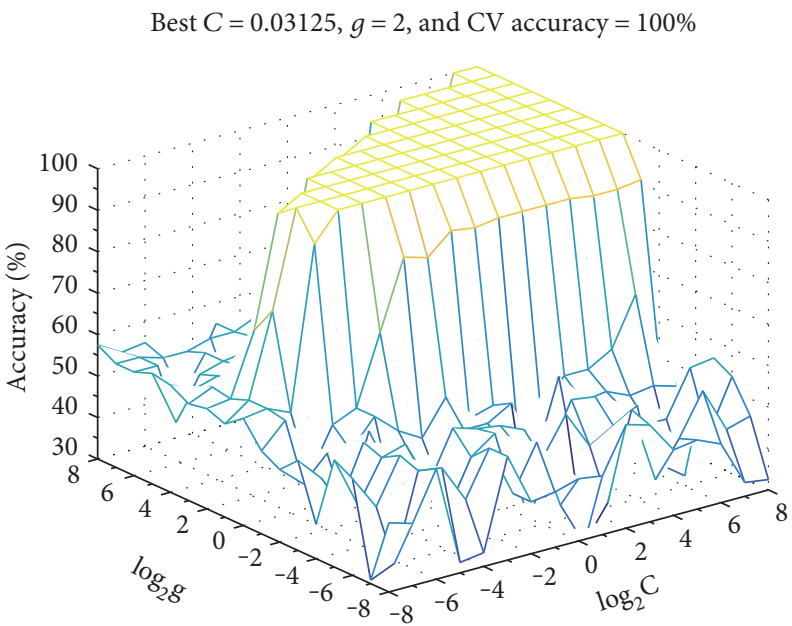

(a)

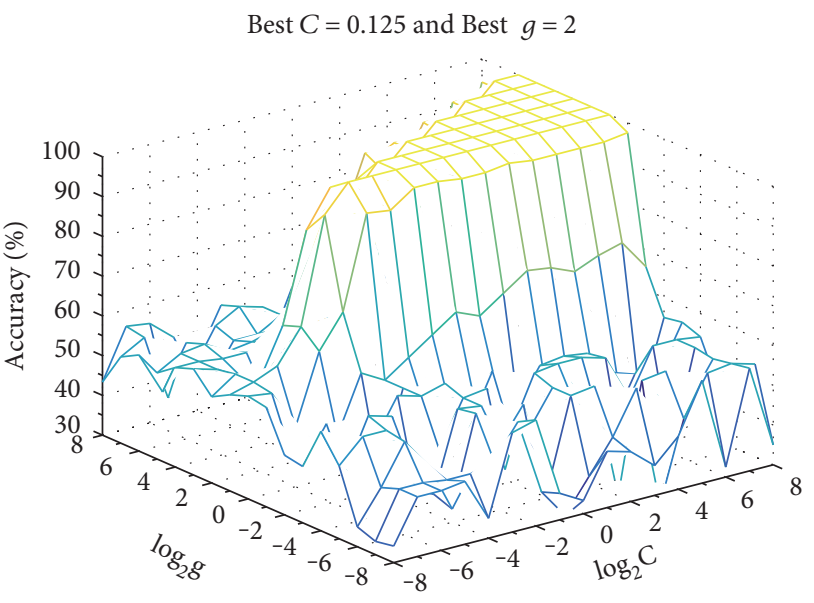

(b)

FIGURE 4: Grid search optimization parameter selection results of a 3D chart. (a) Carbendazim and polyethylene mixture. (b) Carbendazim and rice powder mixture.

TABle 1: Performance of qualitative analyses.

\begin{tabular}{lccc}
\hline Mixture type & $\begin{array}{c}\text { ACCCV } \\
(\%)\end{array}$ & $\begin{array}{c}\text { ACCTR } \\
(\%)\end{array}$ & $\begin{array}{c}\text { ACCTE } \\
(\%)\end{array}$ \\
\hline $\begin{array}{l}\text { Carbendazim and } \\
\text { polyethylene }\end{array}$ & 100 & 100 & 100 \\
$\begin{array}{l}\text { Carbendazim and } \\
\text { rice powder }\end{array}$ & 100 & 100 & 100 \\
\hline
\end{tabular}

peaks at 1.15 and $1.35 \mathrm{THz}$. These absorption peaks can be regarded as the feature of carbendazim.

To detect carbendazim in mixtures, we mixed carbendazim with polyethylene and, later, rice powder. All the mixtures were wrapped in paper, when they were measured by THz-TDS. Figure 3(a) displays the absorption spectra of mixtures with different weight ratios from $0 \%$ to $100 \% \mathrm{w} / \mathrm{w}$. The mixture $0 \% w / w$ is pure polyethylene, and the mixture $100 \%$
TABLE 2: Samples in training set and testing set.

\begin{tabular}{lcc}
\hline Mixture type & $\begin{array}{c}\text { Samples in } \\
\text { training set }\end{array}$ & $\begin{array}{c}\text { Samples in } \\
\text { testing set }\end{array}$ \\
\hline Carbendazim and polyethylene & 166 & 84 \\
Carbendazim and rice powder & 150 & 75 \\
\hline
\end{tabular}

$w / w$ is pure carbendazim. It shows that these absorbance spectra crossed together, and no obvious correlation between absorbance and weight ratio is observed, except for the region of 1.1-1.2 THz. In the region of $1.1-1.2 \mathrm{THz}$, the absorbance spectrum increases with the weight ratio increasing.

Figure 3(b) shows the absorption spectra of mixtures with different weight ratios from $0 \%$ to $100 \% \mathrm{w} / \mathrm{w}$. The mixture $0 \% w / w$ is pure rice powder, and the mixture $100 \% w / w$ is pure carbendazim. The spectral features of mixture with weight ratios ranging from $0 \%$ to $25 \% \mathrm{w} / \mathrm{w}$ are dominated 


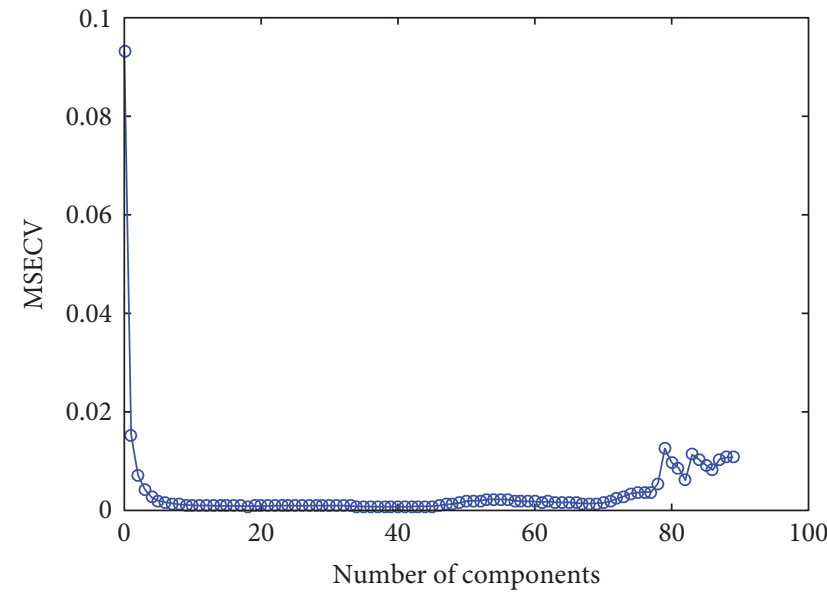

(a)

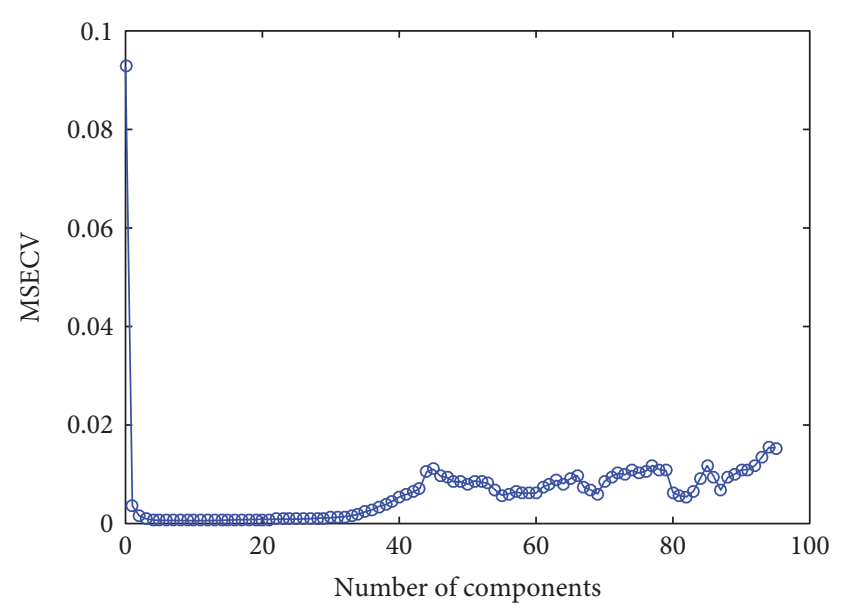

(b)

FIGURE 5: The correlation between the number of components and RMSECV for PLS. (a) Carbendazim and polyethylene mixture. (b) Carbendazim and rice powder mixture.

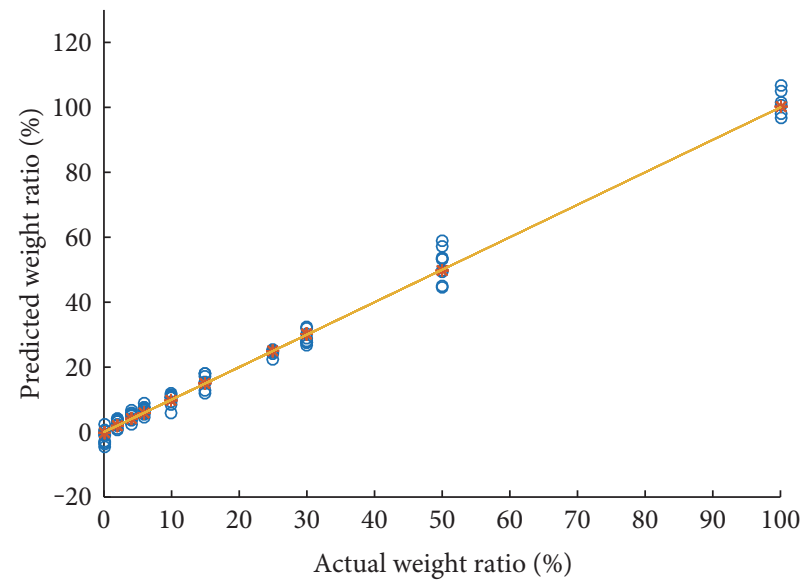

(a)

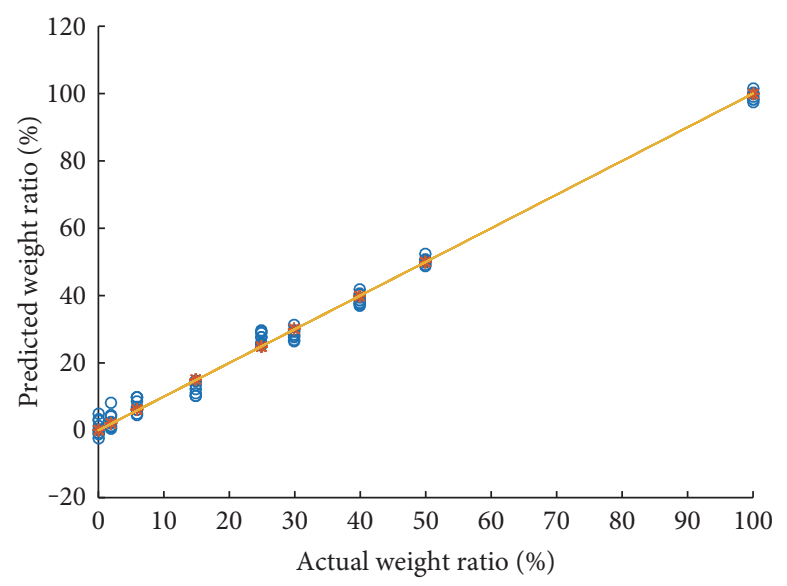

(b)

Figure 6: Performance of PLS. (a) Carbendazim and polyethylene mixture. (b) Carbendazim and rice powder mixture.

by the strong absorbance of rice powder, and no obvious absorption peak at $1.15 \mathrm{THz}$ is observed. However, as the weight ratio is increasing to $30 \% \mathrm{w} / \mathrm{w}$, the peak at $1.15 \mathrm{THz}$ appears. In the region of $1.2-1.4 \mathrm{THz}$, the absorbance spectrum increases with the weight ratio decreasing. Furthermore, an additional absorption peak at $1.23 \mathrm{THz}$ appears. It is caused by rice powder. Further comparing Figures 2, $3(\mathrm{a})$, and 3(b), whether wrapped in paper or not, the absorption spectra of pure carbendazim are the same. It indicates that $\mathrm{THz}$ could penetrate packaging material.

3.2. Qualitative Detection of Carbendazim Mixtures. In Figures 3(a) and 3(b), it illustrates that carbendazim can be easily detected in high weight ratio mixture by absorbance peaks. While as the weight ratio is low, the absorbance of the mixture is similar with pure polyethylene and rice powder. For example, the absorbance of mixture $2 \% \mathrm{w} / \mathrm{w}$ resembles polyethylene in Figure 3(a). The situation is similar in Figure 3(b). It is hard to detect carbendazim in mixtures when the weight ratio is low. To solve this problem, SVM with radial basis function was utilized. As mentioned above, the absorbance of the mixture was used as input of SVM. Grid search technique was selected to find optimal model parameter values, and 10 -fold crossvalidation was implemented to prevent overfitting.

To qualitatively detect carbendazim in low weight ratio mixture (mix carbendazim with polyethylene), we selected 50 samples ( 25 pure polyethylene and 25 mixture $2 \% \mathrm{w} / \mathrm{w}$ ). These samples were divided into a training set and a testing set. In the training set, the number of pure polyethylene and mixture $2 \% w / w$ were 18 and 15 , respectively. At the same time, in the testing set, the number of pure polyethylene and mixture $2 \% w / w$ were 7 and 10 , respectively. Penalty factor $C$ and kernel parameter $g$ are of great effect on SVM after select RBF. The grid search is used to optimize the two parameters $C$ and $g$ based on synchronous optimization idea. The searching interval was $C \in\left[2^{-8}, 2^{8}\right]$ and $g \in\left[2^{-8}, 2^{8}\right]$. Figure 4(a) displays the process of SVM parameter 


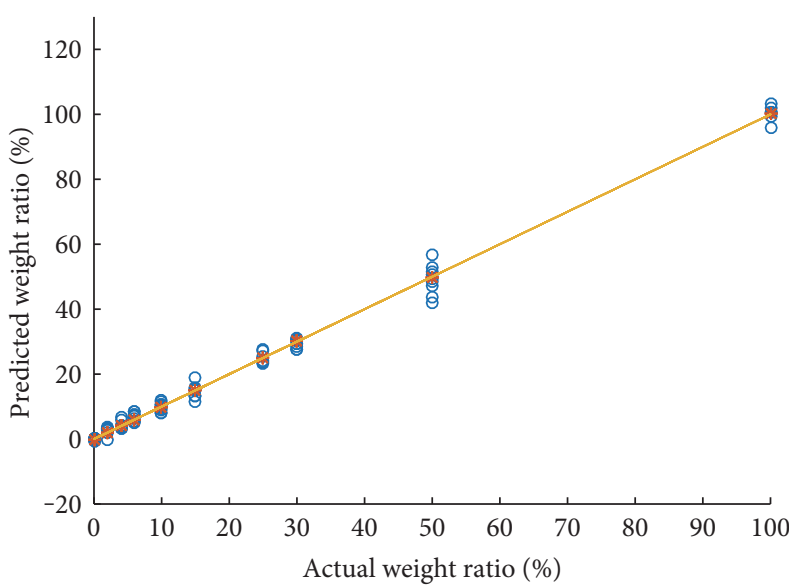

(a)

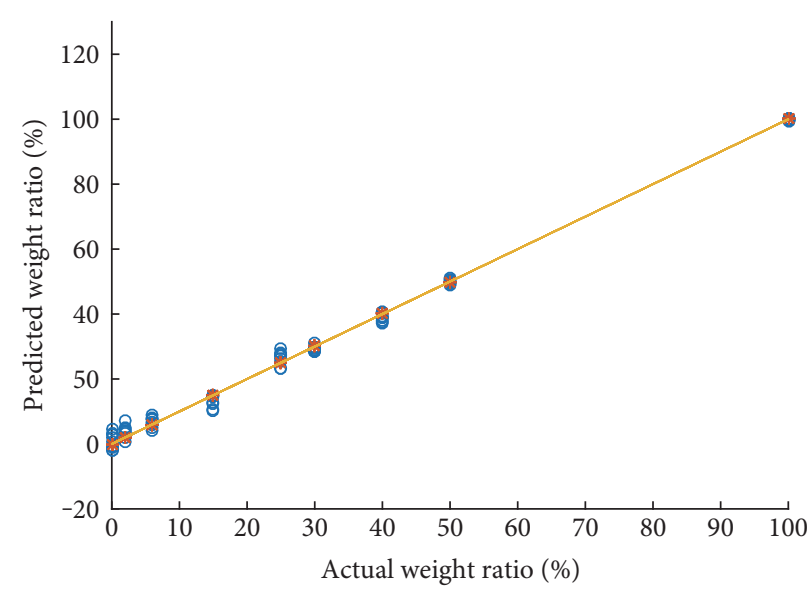

(b)

FIGURE 7: Performance of SVR model. (a) Carbendazim and polyethylene mixture. (b) Carbendazim and rice powder mixture.

TABLE 3: Performance of PLS and SVR in carbendazim and polyethylene mixture.

\begin{tabular}{lcccc}
\hline \multirow{2}{*}{ Model } & Cross validation set & Training set & \multicolumn{2}{c}{ Testing set } \\
& RMSECV & RMSEC & RMSEP & R \\
\hline PLS & 0.0271 & 0.0172 & 0.0255 & 0.9957 \\
SVR & $\mathbf{0 . 0 2 1 8}$ & $\mathbf{0 . 0 0 8 7}$ & $\mathbf{0 . 0 2}$ & $\mathbf{0 . 9 9 7 2}$ \\
\hline
\end{tabular}

optimization. It shows that accuracy depends on penalty factor $C$ and kernel parameter g. When the $C$ is large, a higher possibility of high accuracy is achieved. However, it should be noticed that if $C$ is too large, it will be likely to be overfitting. So, we make a tradeoff between $C$ and accuracy. At last, $C$ was equal to 0.03125 and $g$ was equal to 2 .

Using the same method, we identify mixture $2 \% w / w$ and rice powder. The searching interval was $C \in\left[2^{-8}, 2^{8}\right]$ and $g \in\left[2^{-8}, 2^{8}\right]$. The process of SVM parameter optimization is illustrated in Figure 4(b). As the result, $C$ was equal to 0.125 and $g$ was equal to 2 . Table 1 shows the performance of detecting carbendazim in low weight ratio mixture. It indicates that carbendazim in low weight ratio mixture can be qualitatively detected successfully with the help of SVM.

3.3. Quantitative Detection of Carbendazim Mixtures. Two kinds of mixtures were, respectively, divided into training set and testing set, and the detail was given in Table 2 . PLS and SVR were used for quantificationally detecting carbendazim in mixtures. The absorbance of the mixture was used as input of PLS and SVR. In PLS, 10-fold crossvalidation was implemented to prevent overfitting and select the number of components. In SVR, RBF was utilized, 10 -fold crossvalidation was applied to prevent overfitting, and grid search technique was selected to find the optimal model parameter values.

Figures 5(a) and 5(b) display the correlation between the number of components and RMSECV for PLS. It turned out that MSECV decreased at the beginning and then became worse with increasing the number of components, which are
TABLE 4: Performance of PLS and SVR in carbendazim and rice powder mixture.

\begin{tabular}{lcccc}
\hline Model & Cross validation set & Training set & \multicolumn{2}{c}{ Testing set } \\
& RMSECV & RMSEC & RMSEP & R \\
\hline PLS & 0.0241 & 0.0189 & 0.023 & 0.9967 \\
SVR & $\mathbf{0 . 0 2 2 4}$ & $\mathbf{0 . 0 1 3}$ & $\mathbf{0 . 0 1 8 8}$ & $\mathbf{0 . 9 9 7 8}$ \\
\hline
\end{tabular}

due to introduced noise. In these two kinds of mixtures, the minimum value of MSECV was $7.4 \times 10^{-4}$ and $5.87 \times 10^{-4}$, and the corresponding components were 41 and 11 , accounting for $46.1 \%$ and $11.6 \%$ of the full spectral data of 89 and 95 wavelengths. As the result, the number of components was selected 41 and 11, respectively.

Figures 6(a) and 6(b) show the scattered plot of the predicted weight ratio in PLS against the actual values. Figures 7(a) and 7(b) show the scattered plot of the predicted weight ratio in SVR against the actual values. The reference line represents zero residuals between the predicted and actual values. The closer the scattered points go to the reference line, the better the performance will be.

Comparing Figures 6 and 7, all the predicted points of SVR are closer to the reference line than PLS. It intuitively depicts that SVR is preferable to PLS in the experiment. Tables 3 and 4 display the performance of PLS and SVR. The result on mixtures constituted carbendazim and rice powder shows better performance than the corresponding result on mixtures constituted carbendazim and polyethylene. Comparing the two results, SVR proves to be better than PLS, with relatively larger $R$ and lower RMSEC, RMSECV, and RMSEP sets.

\section{Conclusions}

The applicability of THz-TDS to detect carbendazim in the mixture was present in this study. Unlike previous reports of THz-TDS for detecting pesticide, this work focused on detecting pesticide residue in packaged foods. 
All the mixtures were wrapped in paper, when they were measured. Carbendazim and rice powder revealed their own distinct absorption peaks, while polyethylene had no distinct absorption peaks. Pure carbendazim, polyethylene, and rice powder could be directly identified by their absorption spectra. But, when the weight ratio was low, the absorbance of the mixture is similar with pure polyethylene and rice powder. With the help of SVM, carbendazim could be qualitatively detected in low weight ratio mixture successfully. Moreover, carbendazim was quantificationally detected with PLS and SVR. The results showed that the performance of SVR was better than that of PLS. The best result of SVR is that $R$, RMSECV set, RMSEC set, and RMSEP set were $0.9978,2.24 \%, 1.3 \%$, and $1.8 \%$, respectively.

Despite the limited sensitivity of the current technique, the current work demonstrates its ability to qualitatively and quantitatively detect pesticide residue in packaged foods. Further study will be undertaken to improve the precision and robustness of the prediction model. At last, we expect that THz-TDS become a rapid detection tool for commercial applications in food quality control.

\section{Conflicts of Interest}

The authors declare that there is no conflict of interest regarding the publication of this paper.

\section{Acknowledgments}

This work was supported by the Yulin Normal University Research Grant (Grant no. 2016YJKY06), National Natural Science Foundation of China (Grant no. 21565028), and Guangxi Colleges and Universities Program of Innovative Research Team and Outstanding Talent.

\section{References}

[1] S. H. Baek, J. H. Kang, Y. H. Hwang, K. M. Ok, K. Kwak, and H. S. Chun, "Detection of methomyl, a carbamate insecticide, in food matrices using terahertz time-domain spectroscopy," Journal of Infrared Millimeter and Terahertz Waves, vol. 37, pp. 486-497, 2016.

[2] Y. Hua and H. Zhang, "Qualitative and quantitative detection of pesticides with terahertz time-domain spectroscopy," IEEE Transactions on Microwave Theory and Techniques, vol. 58, pp. 2064-2070, 2010.

[3] Z. Chen, Z. Zhang, R. Zhu, Y. Xiang, Y. Yang, and P. B. Harrington, "Application of terahertz time-domain spectroscopy combined with chemometrics to quantitative analysis of imidacloprid in rice samples," Journal of Quantitative Spectroscopy \& Radiative Transfer, vol. 167, pp. 1-9, 2015.

[4] C. Chafer-Pericas, A. Maquieira, and R. Puchades, "Fast screening methods to detect antibiotic residues in food samples," Tractrends in Analytical Chemistry, vol. 29, pp. 1038-1049, 2010.

[5] R. Martinez, E. Gonzalo, M. Moran, and J. Mendez, "Sensitive method for the determination of organophosphorus pesticides in fruits and surface waters by high-performance liquid chromatography with ultraviolet detection," Journal of Chromatography, vol. 607, pp. 37-45, 1992.
[6] M. Khanmohammadi, S. Armenta, S. Garrigues, and M. de la Guardia, "Mid- and near-infrared determination of metribuzin in agrochemicals," Vibrational Spectroscopy, vol. 46, pp. 82-88, 2008.

[7] S. Armenta, S. Garrigues, and M. de la Guardia, "Determination of iprodione in agrochemicals by infrared and Raman spectrometry," Analytical and Bioanalytical Chemistry, vol. 387, pp. 2887-2894, 2007.

[8] B. Ferguson and X. Zhang, "Materials for terahertz science and technology," Nature Materials, vol. 1, pp. 26-33, 2002.

[9] P. U. Jepsen, D. G. Cooke, and M. Koch, "Terahertz spectroscopy and imaging - modern techniques and applications," Laser \& Photonics Reviews, vol. 5, pp. 124-166, 2011.

[10] F. Rong, L. Zhe, J. Biao-bing et al., "A study of vibrational spectra of L-, D-, DL-alanine in terahertz domain," Spectroscopy and Spectral Analysis, vol. 30, pp. 2023-2026, 2010.

[11] M. D. King, W. D. Buchanan, and T. M. Korter, "Identification and quantification of polymorphism in the pharmaceutical compound diclofenac acid by terahertz spectroscopy and solid-state density functional theory," Analytical Chemistry, vol. 83, pp. 3786-3792, 2011.

[12] D. G. Allis and T. M. Korter, "Theoretical analysis of the terahertz spectrum of the high explosive PETN," Chemphyschem, vol. 7, pp. 2398-2408, 2006.

[13] M. T. Ruggiero and J. A. Zeitler, "Resolving the origins of crystalline anharmonicity using terahertz time-domain spectroscopy and ab initio simulations," Journal of Physical Chemistry B, vol. 120, pp. 11733-11739, 2016.

[14] C. Bing-Hua, M. Guang-Xin, and Z. Ze-Kui, "Terahertz time-domain spectroscopy of dimethoate," Chinese Journal of Analytical Chemistry, vol. 36, pp. 623-626, 2008.

[15] Y. Hua, H. Zhang, and H. Zhou, "Quantitative determination of cyfluthrin in N-hexane by terahertz time-domain spectroscopy with chemometrics methods," IEEE Transactions on Instrumentation and Measurement, vol. 59, pp. 1414-1423, 2010.

[16] W. Qiang and M. Ye-hao, "Qualitative and quantitative identification of nitrofen in terahertz region," Chemometrics and Intelligent Laboratory Systems, vol. 127, pp. 43-48, 2013.

[17] J. B. Sleiman, B. Bousquet, N. Palka, and P. Mounaix, "Quantitative analysis of hexahydro-1,3,5-trinitro-1,3,5, triazine/pentaerythritol tetranitrate (RDX -PETN) mixtures by terahertz time domain spectroscopy," Applied Spectroscopy, vol. 69, pp. 1464-1471, 2015.

[18] C. Burges, "A tutorial on support vector machines for pattern recognition," Data Mining and Knowledge Discovery, vol. 2, pp. 121-167, 1998.

[19] B. Scholkopf, K. Sung, C. Burges et al., "Comparing support vector machines with Gaussian kernels to radial basis function classifiers," IEEE Transactions on Signal Processing, vol. 45, pp. 2758-2765, 1997.

[20] S. Wold, M. Sjostrom, and L. Eriksson, "PLS-regression: a basic tool of chemometrics," Chemometrics and Intelligent Laboratory Systems, vol. 58, pp. 109-130, 2001, International Symposium on Partial Least Squares (PLS 99), Jouy En Josas, France, October, 1999.

[21] N. Sorol, E. Arancibia, S. A. Bortolato, and A. C. Olivieri, "Visible/near infrared-partial least-squares analysis of Brix in sugar cane juice: a test field for variable selection methods," Chemometrics and Intelligent Laboratory Systems, vol. 102, pp. 100-109, 2010. 
[22] H. Drucker, C. Burges, L. Kaufman, A. Smola, and V. Vapnik, "Support vector regression machines," in Advances in Neural Information Processing Systems 9: Proceedings of the 1996 Conference, vol. 9 of Advances in Neural Information Processing Systems, NIPS Fdn, 1997, pp. 155-161, Denver, CO, December, 1996, 10th Annual Conference on Neural Information Processing Systems (NIPS).

[23] V. Vapnik, S. Golowich, and A. Smola, "Support vector method for function approximation, regression estimation, and signal processing," in Advances in Neural Information Processing Systems 9: Proceedings of the 1996 Conference, vol. 9 of Advances in Neural Information Processing Systems, NIPS Fdn, 1997, pp. 281-287, Denver, CO, December, 1996, 10th Annual Conference on Neural Information Processing Systems (NIPS). 

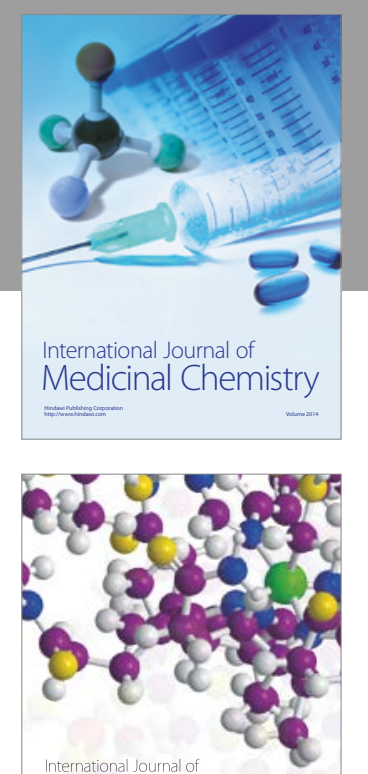

Carbohydrate Chemistry

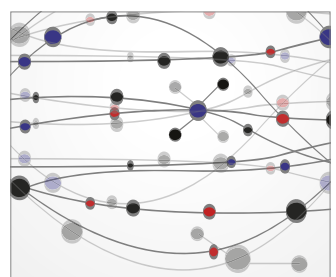

The Scientific World Journal
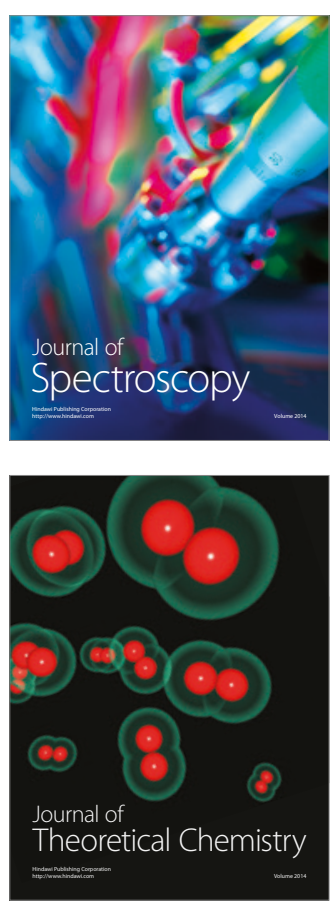
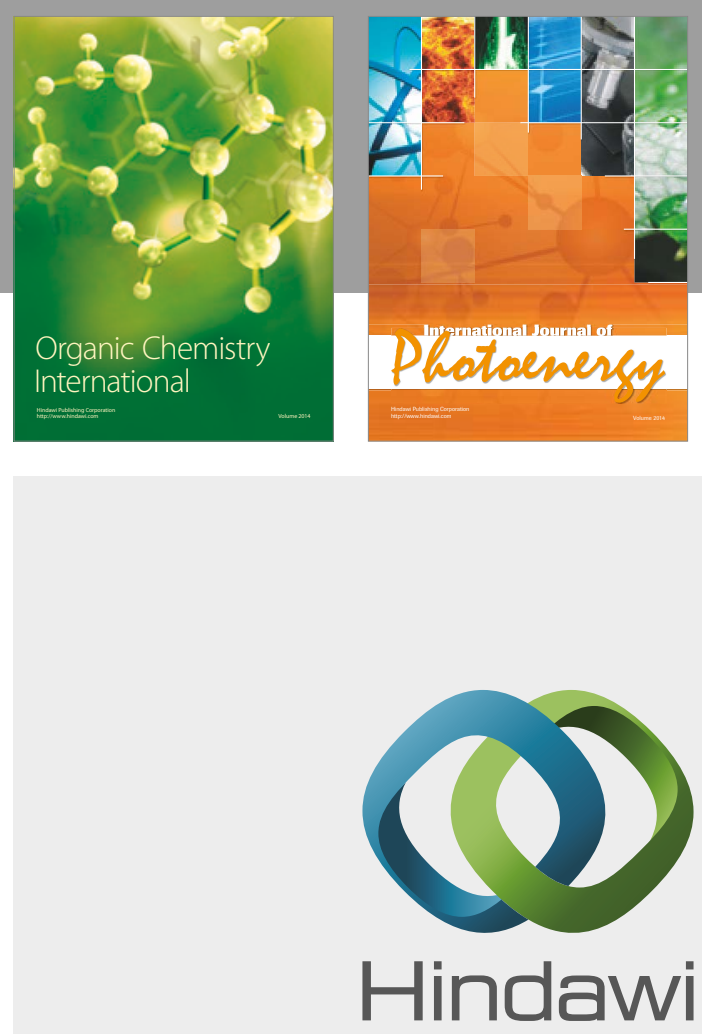

Submit your manuscripts at

https://www.hindawi.com

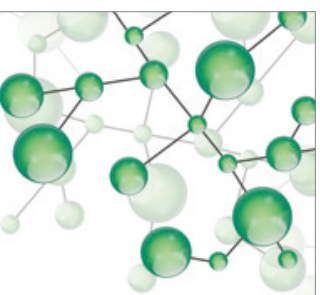

International Journal of

Inorganic Chemistry

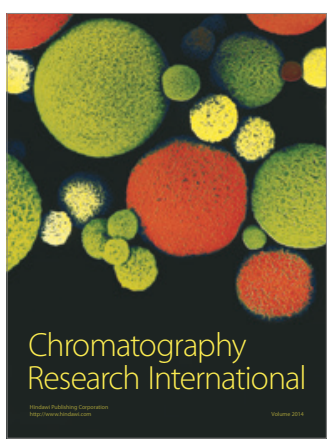

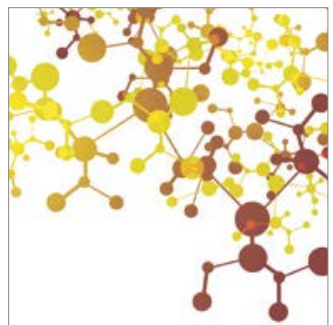

Applied Chemistry
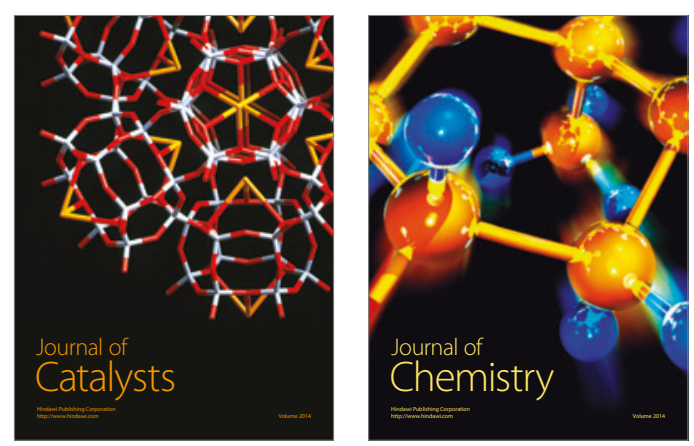
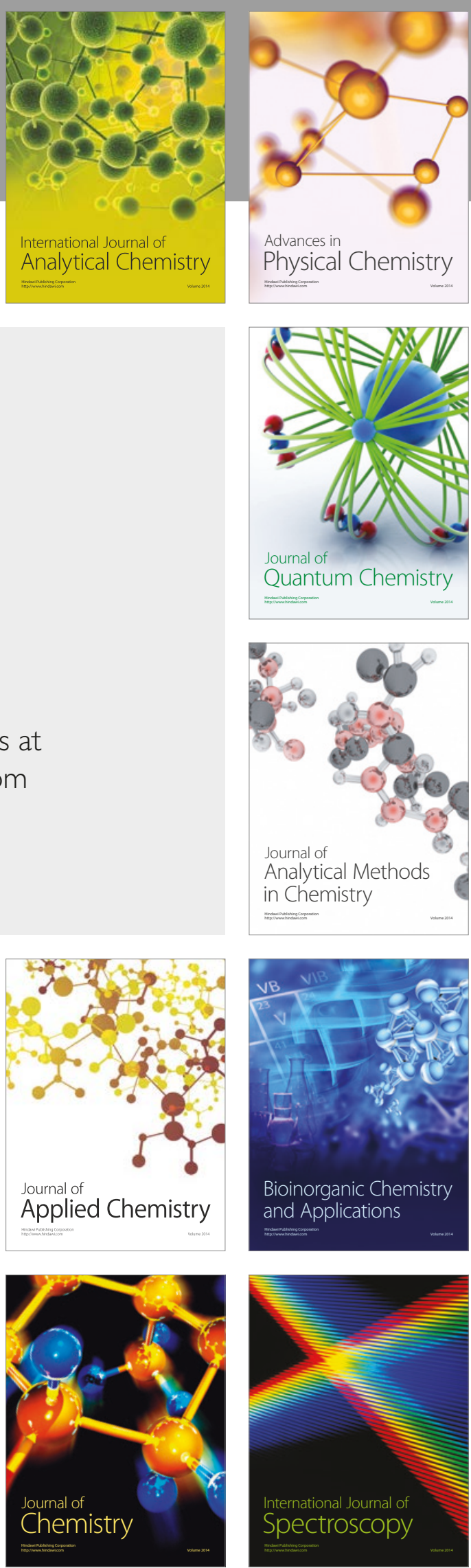